\title{
Candidate to Receive Cardiac Transplant
}

National Cancer Institute

\section{Source}

National Cancer Institute. Candidate to Receive Cardiac Transplant. NCI Thesaurus. Code C99919.

The intended recipient of a heart transplant. (ACC) 